\title{
Universiteit
}

Leiden

The Netherlands

\section{Hall effect measurements on strained and unstrained thin films of} La0.7Ca0.3MnO3 and La0.7Sr0.3MnO3

Dildar, J.M.; Beekman, C.; He, X.; Aarts, J.

\section{Citation}

Dildar, J. M., Beekman, C., He, X., \& Aarts, J. (2012). Hall effect measurements on strained and unstrained thin films of La0.7Ca0.3MnO3 and La0.7Sr0.3MnO3. Physical Review B, 85, 205103. doi:10.1103/PhysRevB.85.205103

Version: $\quad$ Not Applicable (or Unknown)

License: $\quad$ Leiden University Non-exclusive license

Downloaded from: https://hdl.handle.net/1887/45119

Note: To cite this publication please use the final published version (if applicable). 


\title{
Hall effect measurements on strained and unstrained thin films of $\mathrm{La}_{0.7} \mathrm{Ca}_{0.3} \mathrm{MnO}_{3}$ and $\mathrm{La}_{0.7} \mathrm{Sr}_{0.3} \mathrm{MnO}_{3}$
}

\author{
I. Mubeen Dildar, ${ }^{1, *}$ C. Beekman, ${ }^{1, \dagger}$ Xin He, ${ }^{2}$ and J. Aarts ${ }^{1, \ddagger}$ \\ ${ }^{1}$ Kamerlingh Onnes Laboratorium, Leiden University, The Netherlands \\ ${ }^{2}$ Shenyang National Laboratory for Materials Science, Institute of Metals Research, 72 Wenhua Road, Shenyang 110016, \\ People's Republic of China
}

(Received 13 April 2011; revised manuscript received 29 December 2011; published 3 May 2012)

\begin{abstract}
We have studied the Hall effect in thin films of $\mathrm{La}_{0.7} \mathrm{Ca}_{0.3} \mathrm{MnO}_{3}$ and $\mathrm{La}_{0.7} \mathrm{Sr}_{0.3} \mathrm{MnO}_{3}$ deposited on $\mathrm{SrTiO}_{3}$ (STO), $\mathrm{NdGaO}_{3}$ (NGO), and $\mathrm{LaSrGaO}_{3}$ (LSGO) substrates in a temperature range from below (10 $\mathrm{K}$ ) to above $(400 \mathrm{~K})$ the metal-insulator transition, in magnetic fields up to $9 \mathrm{~T}$, and for thicknesses between 7 and $75 \mathrm{~nm}$. The charge-carrier density as calculated from the Hall voltage in a single-band picture shows bulklike values for the thick films, but a significant decrease in thin films (below $20 \mathrm{~nm}$ ), both for strained thin films (on STO) and unstrained thin films on NGO, although less in the case of LSGO. It is well known, however, that a single-band model is not appropriate for the manganates, in which both electron and hole surfaces occur simultaneously. We therefore analyzed the data in a two-band scenario. We still come to the conclusion that the carrier density in the thin films, both strained and unstrained, is lower than in the thicker bulklike films. We discuss this in terms of charge discontinuities at the various interfaces, which appear to play a significant role.
\end{abstract}

DOI: 10.1103/PhysRevB.85.205103

PACS number(s): 75.47.Gk, 72.15.Gd, 71.70.Fk

\section{INTRODUCTION}

Doped manganese perovskite oxides have generated much interest in the last decade, because of the rich physics resulting from the interplay between the electron, lattice, and spin degrees of freedom, and leading to phenomena such as colossal magnetoresistance, phase separation, and full spin polarization. ${ }^{1-3}$ Considering the La-based 1-1-3 family of manganites, the parent compound $\mathrm{LaMnO}_{3}$ has a structure consisting of six corner-sharing $\mathrm{MnO}_{6}$ octahedra on a simple cubic lattice, encaging the La ion. It is an antiferromagnetic Mott insulator which can be driven to a metal by partial substitution of divalent $\mathrm{Sr}^{2+}$ or $\mathrm{Ca}^{2+}$ ions on the $\mathrm{La}^{3+}$ sites. The substitution creates holes as charge carriers and above a critical composition of $x_{c}=0.17$, a ferromagnetic metallic state forms below the Curie temperature $T_{C}$. At a doping level of $x=0.3, T_{C}$ is around $250 \mathrm{~K}$ for $\mathrm{La}_{0.7} \mathrm{Ca}_{0.3} \mathrm{MnO}_{3}(\mathrm{LCMO}$ hereafter) and $370 \mathrm{~K}$ for $\mathrm{La}_{0.7} \mathrm{Sr}_{0.3} \mathrm{MnO}_{3}$ (LSMO). Above $T_{C}$ the material is a polaronic insulator, and the transition at $T_{C}$ is therefore both metal to insulator (MI) and ferromagnetic to paramagnetic. The transition is mainly determined by the competition between the trapping of electrons in Jahn-Teller distortions ${ }^{4}$ and the itinerancy of charge carriers through the double exchange mechanism. ${ }^{5,6}$ The sensitivity of the properties of the manganites to lattice distortions is seen in the effects of hydrostatic pressure, which can significantly enhance $T_{C}$ through rotations of the $\mathrm{MnO}_{6}$ octahedra. $^{7}$

The same sensitivity to lattice distortions makes it possible to apply strain engineering in thin films, by varying the (mis)match between the lattice parameters of film and substrate, as was, for instance, demonstrated in Refs. 8 and 9. For LCMO in particular, the effects of tensile strain are well documented. Growing LCMO with a pseudocubic lattice parameter of $a_{c}=0.387 \mathrm{~nm}$ on $\mathrm{SrTiO}_{3}$ (STO) with $a_{c}=$ $0.391 \mathrm{~nm}$ can lead to a lowering of $T_{C}$ of more than $150 \mathrm{~K}$ for the thinnest films which still show an MI transition. ${ }^{10-12}$ This is generally attributed to the effect of the decrease of the bandwidth of the itinerant $d$ electrons, due to the change in Mn-O-Mn bond angles and the accompanying decrease of the electron hopping parameter, while also the biaxial nature of the strain plays a role. ${ }^{13}$ Such a discussion in terms of the bandwidth of a simple one-band model is not fully correct. Hall effect measurements on single crystals and thick films consistently show, when analyzed in a one-band model, a higher carrier concentration than the chemical doping indicates ( 0.3 holes per unit cell for a $2+$ doping of $30 \%)$. For instance, Asamitsu and Tokura reported a value of a 1.0-hole/Mn site in single crystals of LSMO $(30 \% \mathrm{Sr}) ;{ }^{14} \mathrm{Jacob}$ et al. found a 0.7 -hole/Mn site in thick films of LCMO $(33 \% \mathrm{Ca}) ;^{15}$ and Chun et al. found values up to 2.4 holes per unit cell in single crystals of $\mathrm{La}_{2 / 3}(\mathrm{Ca}, \mathrm{Pb})_{1 / 3} \mathrm{MnO}_{3} \cdot{ }^{16}$ Other reports find similar numbers. ${ }^{17-19}$ More than one band is therefore involved in the transport, and this is also indicated by band-structure calculations, which find Fermi surfaces with both electron and hole character. ${ }^{20}$ Any analysis of Hall data has then to be performed in a scenario of at least two bands, which is not always fully appreciated.

What has not yet been investigated is changes in Hall effect and possibly the carrier density when LSMO or LCMO films become thin and/or strained. This is relevant, for instance, since microscopic mechanisms advocated to explain the decrease of $T_{C}$ in strained thin films do not take a possible change of carrier density into account. Also the possibility of valence variations at the interface would make it possible that the carrier density changes when the films become very thin. Here we present results on the ordinary Hall coefficient measured in high magnetic fields, obtained on such films grown strained on $\mathrm{SrTiO}_{3}$ (STO) and unstrained on $\mathrm{NdGaO}_{3}$ (NGO) and $\mathrm{LaSrGaO}_{3}$ (LSGO). We find that, at low temperatures, the one-band hole density $n_{h, 1}$ for thick films is found close to 1.5 holes per unit cell, similar to the bulk value and demonstrating again that the Hall coefficient is not a measure for the carrier density when analyzed in a one-band scenario. Below a thickness of typically $20 \mathrm{~nm}$ the 

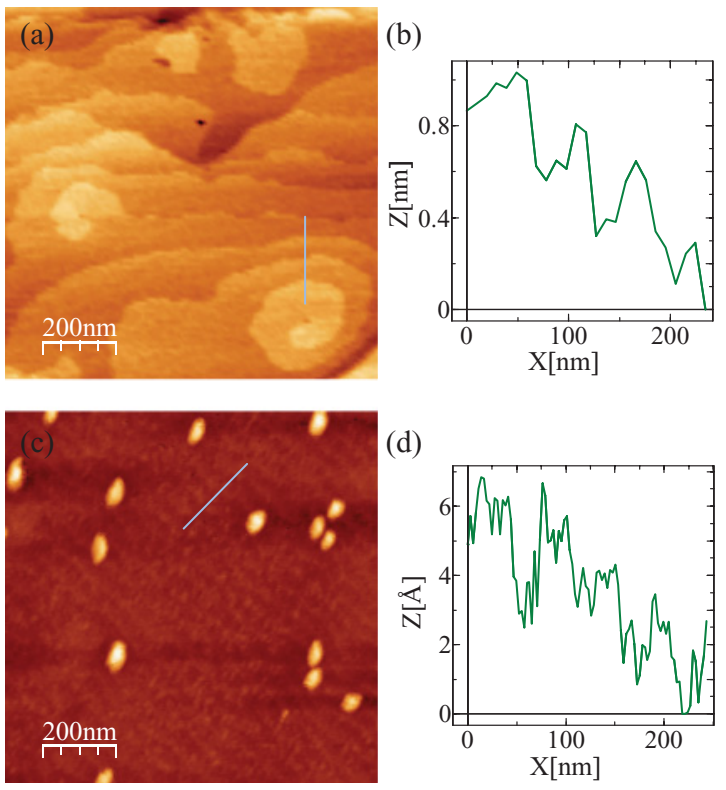

FIG. 1. (Color online) Surface morphology of (a) a 16-nm-thick film of LCMO on STO (called L583); (c) a 25-nm-thick film of LSMO on STO (called LS8). The panels (b) and (d) show the height variation along the lines given in (a) and (c), respectively.

value of $n_{h, 1}$ becomes smaller, and for strained films even goes down to 0.5 . In the one-band model this would mean a decrease of the carrier density, but we show that also in terms of a two-band model (in which the mobilities of the various carriers are separate parameters) the conclusion must be that the thin films have a lower carrier density than thick films or bulk material. We argue that this is in line with observations of dead layers and valence variations at the interface.

\section{EXPERIMENT}

Epitaxial thin films of LCMO $\left(a_{c}=0.3863 \mathrm{~nm}\right)$ and LSMO $\left(a_{c}=0.3873 \mathrm{~nm}\right)$ were deposited on substrates of STO(100) $\left(a_{c}=0.3905 \mathrm{~nm}\right), \mathrm{NGO}(100)\left[a_{c}=0.3851 \mathrm{~nm}\right.$; note that this is $\mathrm{NGO}(110)$ in orthorhombic notation], and LSGO(100) $\left(a_{c}=\right.$ $0.3843 \mathrm{~nm}$ ) using dc sputtering in pure oxygen at a pressure of 3 mbar. The experimental procedure has been described before. $^{12,21,22}$ The films ranged in thickness from 7 to $75 \mathrm{~nm}$ and were characterized by atomic force microscopy (AFM) in tapping mode. Figures 1(a) and 1(b) show morphology and height variation of a 16-nm-thick film of LCMO on STO called L583. The film is flat, and shows step-height variations of the order of a unit cell $(0.4 \mathrm{~nm})$, together with the beginning of spiral growth. Figures 1(c) and 1(d) show the surface morphology and the height variation along a cross section of a 25-nm-thick film of LSMO on STO, called LS8. Again, height variations are not more than $0.4 \mathrm{~nm}$.

The film thickness was measured by $x$-ray reflectivity, using $\mathrm{Cu}-K \alpha$ radiation, where the thickness of the film is defined by period of oscillation. Films grown on STO are strained (mismatch: $-1.07 \%$ in the case of LCMO and $-0.82 \%$ in the case of LSMO), although the strain gradually relaxes with increasing thickness. ${ }^{21}$ Figure 2 shows a reciprocal space map for the 25-nm-thick film LS8 of LSMO on STO around the

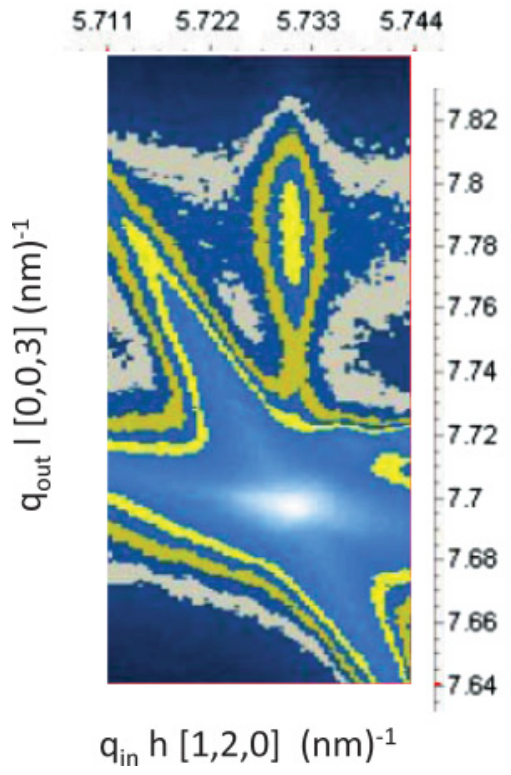

FIG. 2. (Color online) Reciprocal space map of a 25-nm-thick film of LSMO on STO (called LS8), taken around the [123] reflection. The film peak can be seen at $q_{\text {out }} \approx 7.78 \mathrm{~nm}^{-1}$.

[123] reflection. The film is strained and epitaxial. Along the out-of-plane direction a clear film peak is visible separate from the substrate peak, while the in-plane direction shows the same peak values for substrate and film peak. The out-of-plane lattice parameter was determined with this reflection as well as the [002] and [003] reflections, and found to be $0.386 \mathrm{~nm}$, well below the bulk value of $0.389 \mathrm{~nm}$. Films of LCMO with a thickness below $20 \mathrm{~nm}$, grown around the same time as the samples reported on here, showed an out-of-plane lattice parameter around $0.381 \mathrm{~nm}$, much reduced from the pseudocubic bulk value, and confirming that such films are fully strained. Films on NGO (mismatch: less than $0.3 \%$ in the case of LCMO and LSMO) and on LSGO (mismatch: $0.52 \%$ in the case of LCMO and $0.78 \%$ in the case of LSMO) are unstrained. A physical properties measurement system (PPMS, Quantum Design) was used for the temperature and field control. External current sources and voltmeters were used for the transport measurements of unstructured and structured thin films. The samples were patterned photolithograhically into Hall structures. The bridges were $200 \mu \mathrm{m}$ in width and $3.6 \mathrm{~mm}$ in length while the distance between two voltage contacts was $0.6 \mathrm{~mm}$. Argon ion beam etching (etch rate $0.3 \mathrm{~nm} / \mathrm{sec}$ ) was used for structuring the LCMO films, and wet etching $\left(\mathrm{H}_{2} \mathrm{O}: \mathrm{HF}: \mathrm{HCl}: \mathrm{HNO}_{3}=25: 1: 1: 1\right)$ with an etch rate of $2 \mathrm{~nm}$ per second for the LSMO thin films. After Ar etching, the LCMO samples were treated with oxygen plasma in order to restore the insulating properties of the STO substrate. ${ }^{22}$ For the measurements of the Hall coefficient, the temperature was stabilized to better than $20 \mathrm{mK}$. The data were taken at constant temperature, with a current between $1 \mathrm{~mA}$ and $100 \mu \mathrm{A}$. A full current-voltage measurement was made regularly to check linearity and the absence of an offset. The magnetic field (oriented perpendicular to the sample plane) was scanned from $-9 \mathrm{~T}$ to $+9 \mathrm{~T}$, which takes about $3 \mathrm{~h}$. 


\section{RESULTS}

Figure 3(a) shows the temperature dependence of the longitudinal resistance $R(T)$ of structured films of LCMO for different thicknesses on different substrates $(9 \mathrm{~nm}, 16 \mathrm{~nm}$, $75 \mathrm{~nm}$ on STO, $16 \mathrm{~nm}$ on LSGO). Data are given both for zero field, and in a field of $9 \mathrm{~T}$. The behavior is as reported before: the thinnest film on STO shows a peak temperature of the resistance $T_{p}$ around $130 \mathrm{~K}$, which then increases to $200 \mathrm{~K}$ for the 75-nm film. The films on better matching substrates show higher value of $T_{p}$, even though they are very thin. Figure 3(b) shows similar data for LSMO (LS15 with thickness $7 \mathrm{~nm}$, LS8 with thickness $25 \mathrm{~nm}$, a film of $75 \mathrm{~nm}$ ) on STO. The effects of strain on LSMO are less strong, as can be seen from the fact that $T_{p}$ for the $25-\mathrm{nm}$ film is already close to the bulk value. The high-field data show the usual colossal magnetoresistance effect.

Next, the Hall voltage $U_{H}$ was measured on these films as a function of the magnetic field $H_{a}$ applied perpendicular to the plane of the sample, and at different constant temperatures. In all cases the offset voltage was low, except for the case of 9-nm
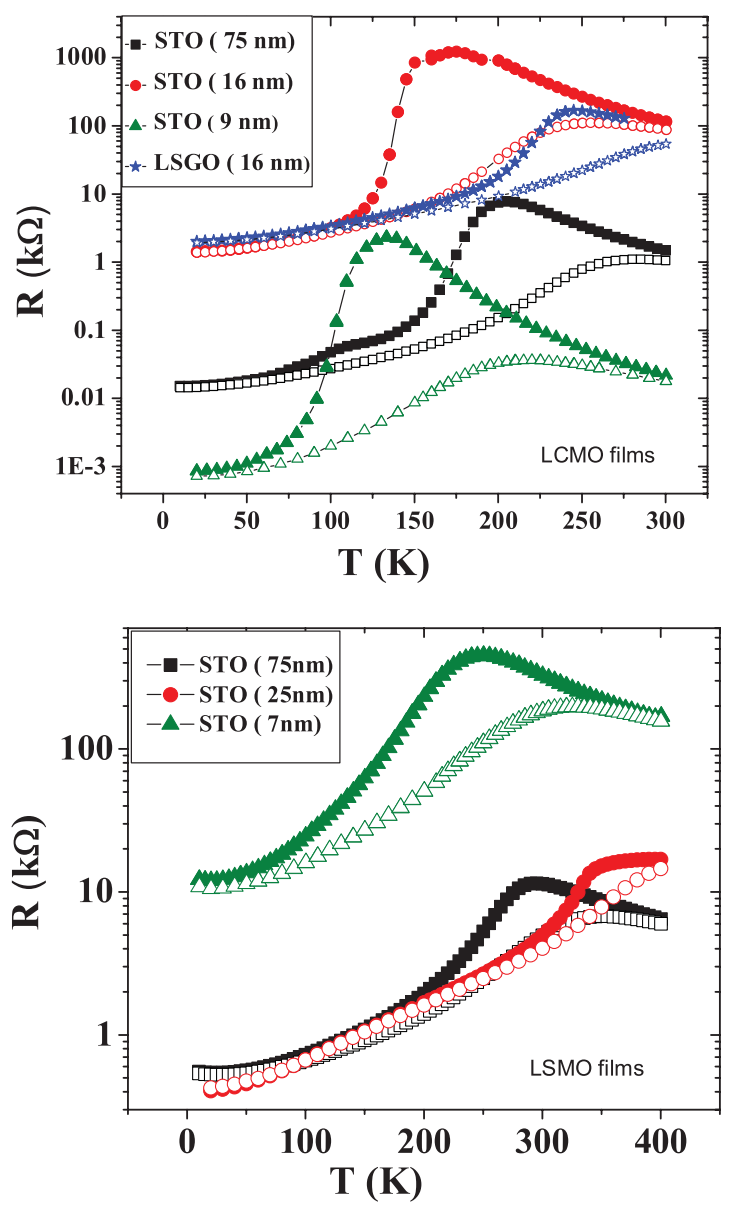

FIG. 3. (Color online) Resistance $R$ versus temperature $T$ in zero field and in a 9-T field of structured films of $\mathrm{La}_{0.7} \mathrm{Ca}_{0.3} \mathrm{MnO}_{3}$ (LCMO) and $\mathrm{La}_{0.7} \mathrm{Sr}_{0.3} \mathrm{MnO}_{3}$ (LSMO) of different thickness on different substrates. (a) LCMO: $9 \mathrm{~nm}, 16 \mathrm{~nm}, 75 \mathrm{~nm}$ on STO, $16 \mathrm{~nm}$ on LSGO. (b) LSMO: $7 \mathrm{~nm}$ (LS15), $25 \mathrm{~nm}$ (LS8), $75 \mathrm{~nm}$ on STO. The filled symbols show 0 -field data, the open symbols show data taken at $9 \mathrm{~T}$.

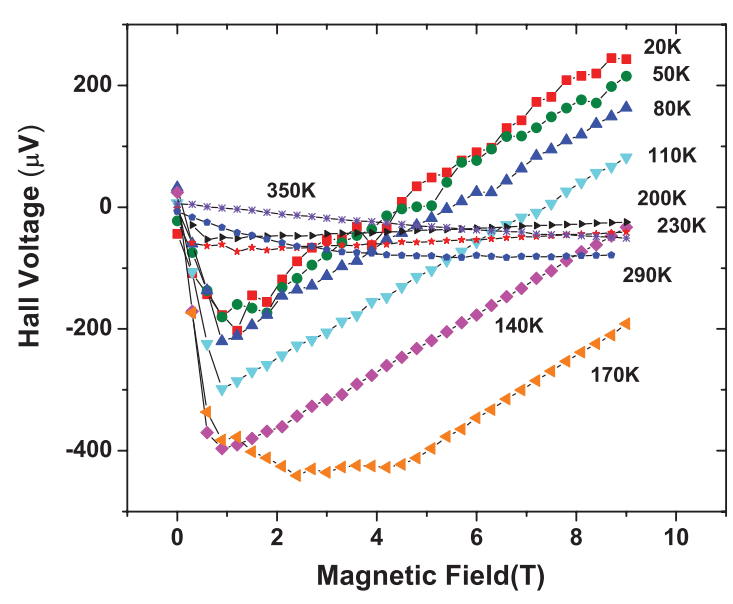

FIG. 4. (Color online) Hall voltage as function of magnetic field at constant temperature (as indicated) for the film of $7 \mathrm{~nm}$ LSMO on STO, called LS15.

LCMO on STO. That sample will not be considered further. Figure 4 shows $U_{H}$ for LS15 (the 7-nm film of LSMO on STO), as determined by taking the average of the measured voltage at $+H_{a}$ and $-H_{a}$. For this film $T_{p}$ is around $230 \mathrm{~K}$. Below $T_{p}, U_{H}$ as a function of increasing field first drops down, and then increases linearly. Around and above $T_{p}$, the crossover to linear behavior becomes less pronounced, and vanishes above $230 \mathrm{~K}$. The behavior below $T_{p}$ is usually understood from the relation

$$
\rho_{H}=\frac{E_{H}}{J}=\frac{U_{H} d_{F}}{I}=\mu_{0}\left(R_{H} H_{a}+R_{A} M\right) .
$$

Here, $E_{H}$ is the electric field, $J$ is the current density, $I$ is the current, $d_{F}$ is the film thickness, $R_{H}$ is the ordinary Hall coefficient, and $R_{A}$ is the anomalous Hall coefficient. That can be due to the magnetization, ${ }^{23}$ but in manganites $R_{A}$ appears to have a different origin, since its contribution grows with increasing temperature right up to the transition temperature. This behavior is well documented ${ }^{15,16,18}$ and was ascribed to the carriers moving in a nontrivial background of core spins. ${ }^{24}$ The positive slope seen in the data of Fig. 4 signifies the hole character of the carriers. The value for $R_{H}$ is determined from the linear part of the data for $U_{H}\left(H_{a}\right)$. For instance, at $20 \mathrm{~K}$ the slope is about $53 \mu \mathrm{V} / \mathrm{T}$ which, with a thickness of $7 \mathrm{~nm}$ and a measurement current of $500 \mu \mathrm{A}$ yields $R_{H}=7.5 \times 10^{-10} \Omega \mathrm{m}$. In a single-band picture, the carrier density directly follows from $n_{c}=V_{\mathrm{uc}} /\left(e R_{H}\right)$, with $V_{\mathrm{uc}}$ the unit-cell volume and $e$ the electron charge. Around $T_{C}$ there is no large linear regime, and there we used the high-field part of the data above roughly $6 \mathrm{~T}$. We are mainly interested in the low-temperature carrier density, but in this way the transition is clearly visible. In Fig. 5 we show a representative set of data taken from different film-substrate combinations of $1 / R_{H}$ and the hole density $n_{h, 1}$ evaluated in a one-band picture. In all cases there is a relatively steep rise around $T_{C}$ of the film, followed by saturated behavior to low temperatures. At low temperatures, the 75-nm LSMO film on STO shows a value of $n_{h, 1} \approx 1.5$, in line with previous reports on thick films. The 16 -nm LCMO film on LSGO has $n_{h, 1} \approx 1.3$, slightly lower than the bulk. The thin strained films deposited on STO show 


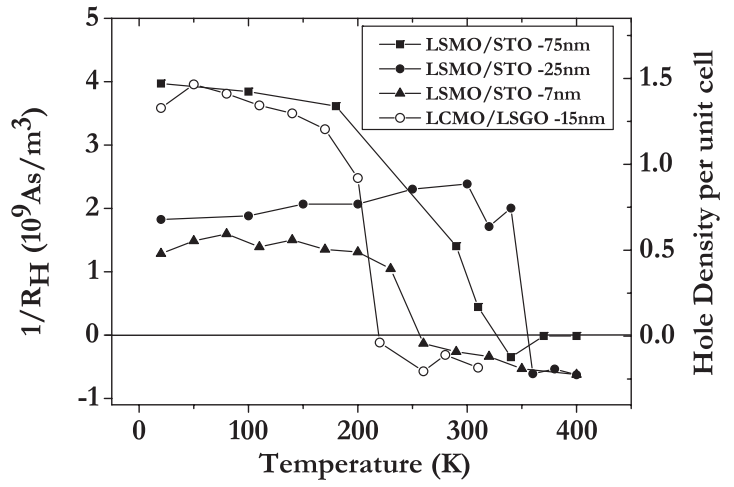

FIG. 5. Inverse Hall resistance $R_{H}$ (left-hand scale) and hole density per unit cell (right-hand scale) as function of temperature, for LSMO films with thicknesses $7 \mathrm{~nm}$ (LS15), $25 \mathrm{~nm}$ (LS8), $75 \mathrm{~nm}$ on STO, and an LCMO film of $15 \mathrm{~nm}$ on LSGO.

values around 0.7. In Fig. 6 the values of $n_{h, 1}$ for all samples are collected, taken at $20 \mathrm{~K}$.

\section{DISCUSSION AND CONCLUSION}

As we noted above, a one-band picture is not appropriate to discuss Hall data. We use the same starting point as done before, namely the band-structure calculations of Pickett and Singh, who found a hole pocket of cubic shape, containing 0.55 holes and centered around the $R$ point of the Brillouin zone (the corner point of a simple cube), and an electron pocket of spherical shape, containing 0.05 electrons and centered around the $\Gamma$ point. ${ }^{20,25}$ This was confirmed by other calculations as well as by several experiments. In particular Livesay et al. reported similar calculations and experimentally verified the presence and shape of both Fermi surfaces (FS) by positron annihilation ${ }^{26}$ on a single crystal of $\mathrm{La}_{0.7} \mathrm{Sr}_{0.3} \mathrm{MnO}_{3}$. Photoemission studies on thin films (40 nm) of $\mathrm{La}_{1-x} \mathrm{Sr}_{x} \mathrm{MnO}_{3}$ grown on NGO confirmed the existence of the $\Gamma$-centered electron pocket for $x=0.3 .^{27}$ This film was strained, and it is important to note that in such films this part of the FS still

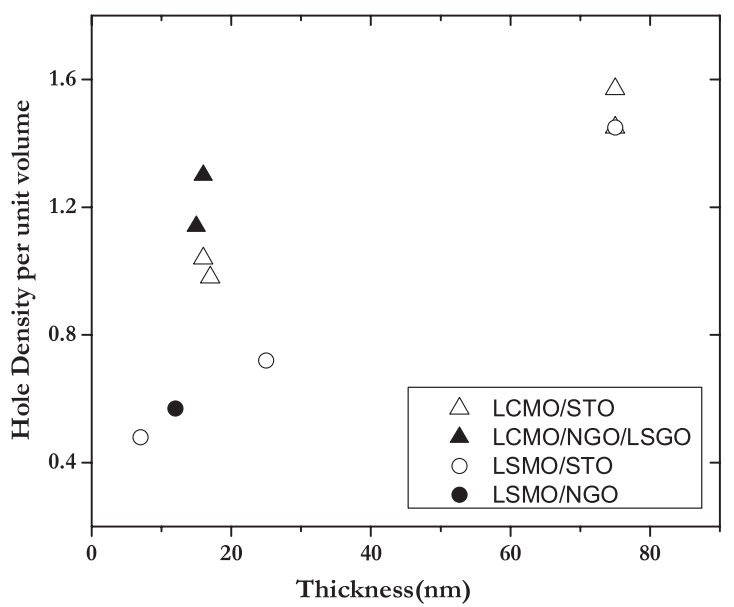

FIG. 6. One-band hole density $n_{h, 1}$ versus film thickness. Filled symbols show films on NGO/LSGO (triangles: LCMO; circles: LSMO), empty symbols show films on STO. exists. In photoemission no clear evidence is found for the hole-type FS, as discussed in Ref. 28.

In the two-band picture, the relation between the measured Hall resistance $R_{H}$ and the two carrier densities is as follows:

$$
R_{H}=\frac{r_{h} n_{h} \mu_{h}^{2}-r_{e} n_{e} \mu_{e}^{2}}{e\left(n_{h} \mu_{h}+n_{e} \mu_{e}\right)^{2}} .
$$

Here, $n_{h, e}$ are the hole, electron densities, $\mu_{h, e}$ are the hole, electron mobilities, and $r_{h, e}$ are factors taking the shape of the FS into account, with $r=1$ for a spherical surface and $r=1 / 2$ for a cubic surface. ${ }^{29}$ The shape factors were used by Chun et al. in their analysis, ${ }^{16}$ but not by Jakob et al..$^{15}$, nor by Bibes et al. ${ }^{30}$ For our analysis, the shape factors turn out to be important.

In order to work with Eq. (2), several assumptions have to be made. Taking the Pickett-Singh carrier densities, we can resolve for the mobility ratio $\mu_{e} / \mu_{h}$ using the measured $R_{H}$. For the three 75-nm LCMO and LSMO films on STO, we find mobilities ratios of 0.8 and 0.9 , which is of the same order of magnitude as found before. ${ }^{16}$ The actual numbers are given in Table I. When we try the same for the data with lower values of $1 / R_{H}$ (and therefore $n_{h}$ ), however, we cannot find reasonable values for $\mu_{e} / \mu_{h}$ which would reproduce the Picket-Singh densities of $0.55\left(n_{h}\right)$ and $0.05\left(n_{e}\right)$. This is shown pictorially in Fig. 7 where we plot the values of $n_{h, 2}$ versus $V_{\mathrm{uc}} /\left(e R_{H}\right)=$ $n_{h, 1}$ for different values of $\mu_{e} / \mu_{h}$.

The figure first shows the solution of Chun et al. for their single crystal, namely a mobility ratio of about 1.5 at a Hall resistance corresponding to $n_{h, 1}=2.4$. It also shows the asymptote which is found when $\mu_{e} / \mu_{h}$ goes to zero, which is the reason that for values below roughly $V_{\mathrm{uc}} /\left(e R_{H}\right)=$ $n_{h, 1}=1.2$, no solution can be found which reproduces the Pickett-Singh carrier densities. For the 75-nm samples, the mobility ratio is seen to be about 1 . For samples with $n_{h, 1}$ below 1.2 we plot them at a mobility ratio of 0.3 , which means we allow for a factor 3 change in the ratio. As can be seen in the figure, this leads to a decrease of $n_{h, 2}$ from 0.55 to about 0.25 . The conclusion from the analysis therefore is that in the two-band model, and using $r=1 / 2$, the data in particular for the thinnest films cannot be described by changing only the mobility ratio, but that the carrier density also has to be lower. This is somewhat different when $r=1$ is used for the hole pocket. Solutions are then possible down to $n_{h, 1} \approx 0.6$. However, the single-crystal experiments of Chun et al. cannot be described in this way, and the spread of mobility ratios which describe our samples becomes large: from 0.3 at $n_{h, 1}=$ 0.6 to 2.5 at $n_{h, 1}=1.5$, roughly an order of magnitude, while the 7-nm LSMO on STO still cannot be described.

Generally, the LSMO films show a significantly decreased carrier density even when the mobility ratio is lowered to 0.3 , while the LCMO films show a smaller decrease, of the order of $10 \%$. However, lowering the value of $\mu_{e} / \mu_{h}$ from 1.5 to 0.3 is not completely physical, because of the following. From the band-structure calculations it was found that the holes are very light ( $m_{h} \approx 0.6 m^{*}, m^{*}$ the bare electron mass), while the electrons are very heavy $\left(m_{h} \approx 14 m^{*}\right)$. A lower $\mu_{e} / \mu_{h}$ would mean a still larger value of $m_{e}$ and/or a still smaller value of $m_{h}$, which seems unlikely. The analysis therefore strongly indicates that for both LSMO and LCMO films the carrier density of films of roughly $20 \mathrm{~nm}$ or less is significantly smaller 
TABLE I. The table shows type of film, type of substrate, film thickness, peak temperature $T_{p}$, the value of $1 / R_{H}$ taken at $20 \mathrm{~K}$, and the corresponding value of the one-band hole density $n_{h, 1}$. It also shows the two-band hole density $n_{h, 2}$ and the mobility ratio $\mu_{e} / \mu_{h}$ determined in the following way: if $n_{h, 2}$ can be fixed to 0.55 , the mobility ratio is a free parameter; if no mobility ratio can be found to match 0.55 , the ratio is fixed at 0.3 .

\begin{tabular}{lcccccc}
\hline \hline & & Thickness & $T_{p}$ & $1 / R_{H}$ & & \\
Film & Substrate & $\mathrm{nm}$ & $\mathrm{K}$ & $10^{9} \mathrm{C} / \mathrm{m}^{3}$ & $n_{h, 1}$ & $n_{h, 2}$ \\
\hline LSMO & STO & 75 & 325 & 4.0 & 1.45 & 0.55 \\
LSMO & STO & 25 & 370 & 2.0 & 0.72 & 0.32 \\
LSMO & STO & 7 & 250 & 1.3 & 0.48 & 0.2 \\
LSMO & NGO & 12 & 370 & 1.6 & 0.57 & 0.25 \\
LCMO & STO & 75 & 190 & 4.0 & 1.45 & 0.55 \\
LCMO & STO & 75 & 210 & 4.3 & 1.57 & 0.55 \\
LCMO & STO & 17 & 175 & 2.7 & 0.98 & 0.3 \\
LCMO & STO & 16 & 175 & 2.85 & 1.04 & 0.4 \\
LCMO & NGO & 15 & 210 & 2.9 & 1.14 & 0.3 \\
LCMO & LSGO & 15 & 245 & 3.7 & 1.3 & 0.3 \\
\hline \hline
\end{tabular}

than in the bulk. The one exception is the 15-nm LCMO film on LSGO, which can be described with parameters still close to the bulk values.

Both in the strained and the unstrained case therefore more carriers are trapped when the films become thin, and this is also the case in the high fields of $9 \mathrm{~T}$ used in the measurements. This makes the trapping a very robust effect. For films on STO, this finding is quite in line with a number of different observations reported in the literature. For LCMO/STO, it was shown by Bibes et al. ${ }^{11}$ from NMR data that a nonferromagnetic insulating phase of about $5 \mathrm{~nm}$ exists close to the interface. For LSMO/STO, recent work showed a nonconducting layer of about $3 \mathrm{~nm}$ at the interface, ${ }^{31}$ which is probably connected to an orbital reconstruction, ${ }^{32}$ accompanied by a slight increase in the amount of $\mathrm{Mn}^{3+}$ ions. ${ }^{33}$ We have similar evidence of $\mathrm{Mn}^{3+}$ enrichment from electron energy loss spectroscopy (EELS) at the interface of LCMO/STO. ${ }^{34}$ The cause of this charge transfer is probably the charge discontinuity which occurs at the LSMO/STO and LCMO/STO interfaces, similar to what

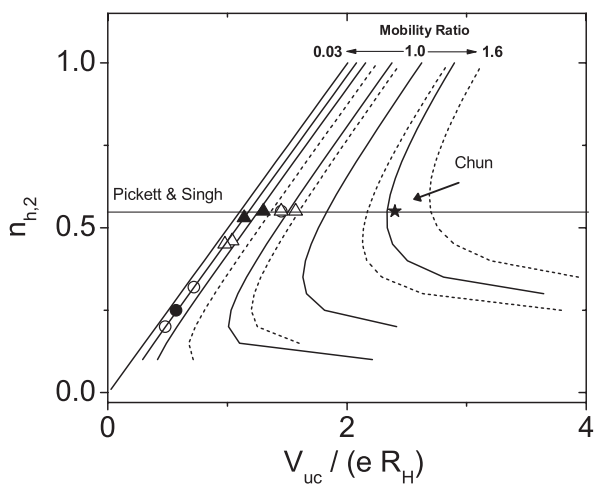

FIG. 7. The two-band hole density $n_{h, 2}$ plotted as function of $V_{\text {uc }} / e R_{H}$, with the contours showing different values of the mobility ratio $\mu_{e} / \mu_{h}$. Contours are given at mobility ratios $0.03,0.1,0.5,0.7$, $0.9,1.0,1.2,1.4,1.5,1.6$. For $V_{\text {uc }} /\left(e R_{H}\right)=n_{h, 1}>1.2$, its value is fixed at the Pickett-Singh value of 0.55 . Below $n_{h, 1}=1.2$, data are plotted on the $\mu_{e} / \mu_{h}=0.3$ contour. Filled symbols show films on NGO/LSGO (triangles: LCMO; circles: LSMO), empty symbols show films on STO. is intensively researched on the $\mathrm{LaAlO}_{3} / \mathrm{SrTiO}_{3}$ interface. ${ }^{35}$ Just as the polar $\mathrm{LaAlO}_{3}$ consists of charged blocks of $(\mathrm{LaO})^{1+}$ and $\left(\mathrm{AlO}_{2}\right)^{1-}$, the LSMO layer consists of blocks $\left[\left(\mathrm{La}_{0.7} \mathrm{Ca}_{0.3} \mathrm{O}_{3}\right]^{0.7+}\right.$ and $\left(\mathrm{MnO}_{2}\right)^{0.7-}$ which cause a charge discontinuity at the interface with STO. The number we extract for the 7-nm LSMO/STO film of a carrier density about half of the bulk value therefore seems entirely reasonable and attributable to the Mn valency changes in the LSMO layer close to the interface.

Looking separately at LCMO and LSMO, we see from Table I that the thinnest films, one on STO and one on NGO, both show significant decrease of carrier density, indicating that the role of strain is less important than the role of the interface. For LCMO the films are not as thin, and the decrease is less (of the order of $20 \%$ ), while the film on LSGO shows much less of a change. Again this would fit the charge discontinuity picture. For NGO the charge of the blocks is $(+1,-1)$. For LSGO it is half that value, because of the mixed $\mathrm{La}, \mathrm{Sr}$ valence, and therefore closest in charge matching to the LSMO layer. The LSGO sbstrate is therefore expected to give the smallest effect. Together, the data indicate that both for the wider and the smaller bandwidth system, strain is a less important issue than the interface, and that the interface effects are not small but of the order of a number of nanometers; larger than what is sometimes assumed on the basis of the expected screening lengths.

In conclusion, we have shown that, even though the inverse Hall constant cannot be directly connected to a carrier density since a one-band picture does not apply, also an analysis in a two-band scenario shows that the carrier density decreases both in LSMO and LCMO films when they become thinner. Strain does not appear to be the main driver. Rather, the data taken with different substrates indicate interface effects, in line with current models for charge discontinuities. The range of these effects appears to be a few nanometers.

\section{ACKNOWLEDGMENTS}

We thank Zicheng Zhong, Mark Golden, and Jeroen van den Brink for discussions on the issue of the band structure, and Sybolt Harkema for performing high-resolution x-ray- 
diffraction measurements. This research was funded through research grants of the Stichting F.O.M. and the K.N.A.W (Netherlands-China program). I.M.D. was supported by the
Higher Education Commission (HEC) of Pakistan and on study leave from the University of Engineering and Technology, Lahore, Pakistan.
* On leave from Dept. of Physics, University of Engineering and Technology, Lahore-54890, Pakistan.

${ }^{\dagger}$ Present address: Department of Physics and Institute of Optical Sciences, University of Toronto, Canada.

†aarts@physics.leidenuniv.nl

${ }^{1}$ E. Dagotto, T. Hotta, and A. Moreo, Phys. Rep. 344, 1 (2001)

${ }^{2}$ M. B. Salamon and M. Jaime, Rev. Mod. Phys. 73, 583 (2001).

${ }^{3}$ Y. Tokura, Rep. Prog. Phys. 69, 797 (2006).

${ }^{4}$ A. J. Millis, Nature (London) 392, 147 (1998).

${ }^{5}$ C. Zener, Phys. Rev. 82, 403 (1951).

${ }^{6}$ P. W. Anderson and H. Hasegawa, Phys. Rev. 100, 67 (1955).

${ }^{7}$ H. Y. Hwang, T. T. M. Palstra, S.-W. Cheong, and B. Batlogg, Phys. Rev. B 52, 15046 (1995).

${ }^{8}$ Y. Konishi, Z. Fang. M. Izumi, T. Mamako. M. Kasai, H. Kuwahara, M. kawasaki, K. Terakura and Y. Tokura, J. Phys. Soc. Jpn. 68, 3790 (1999).

${ }^{9}$ Z. Fang, I. V. Solovyev, and K. Terakura, Phys. Rev. Lett. 84, 3169 (2000).

${ }^{10}$ J. Aarts, S. Freisem, R. Hendrikx, and H. W. Zandbergen, Appl. Phys. Lett. 72, 2975 (1998).

${ }^{11}$ M. Bibes, L1. Balcells, S. Valencia, J. Fontcuberta, M. Wojcik, E. Jedryka, and S. Nadolski, Phys. Rev. Lett. 87, 067210 (2001).

${ }^{12}$ Z. Q. Yang, R. Hendrikx, J. Aarts, Y. L. Qin, and H. W. Zandbergen, Phys. Rev. B 70, 174111 (2004).

${ }^{13}$ A. J. Millis, T. Darling, and A. Migliori, J. Appl. Phys. 83, 1588 (1998).

${ }^{14}$ A. Asamitsu and Y. Tokura, Phys. Rev. B 58, 47 (1998).

${ }^{15}$ G. Jakob, F. Martin, W. Westerburg, and H. Adrian, Phys. Rev. B 57, 10252 (1998).

${ }^{16}$ S. H. Chun, M. B. Salamon, and P. D. Han, J. Appl. Phys. 85, 5573 (1999).

${ }^{17}$ G. J. Snyder, R. Hiskes, S. DiCarolis, M. R. Beasley, and T. H. Geballe, Phys. Rev. B 53, 14434 (1996).

${ }^{18}$ P. Matl, N. P. Ong, Y. F. Yan, Y. Q. Li, D. Studebaker, T. Baum, and G. Doubinina, Phys. Rev. B 57, 10248 (1998).
${ }^{19}$ N. G. Bebenin, R. I. Zainullina, N. S. Bannikova, V. V. Ustinov, and Ya. M. Mukovskii, Phys. Rev. B 78, 064415 (2008).

${ }^{20}$ W. E. Pickett and D. J. Singh, Phys. Rev. B 55, R8642 (1997).

${ }^{21}$ Z. Q. Yang, R. Hendrikx, J. Aarts, Y. Qin, and H. W. Zandbergen, Phys. Rev. B 67, 024408 (2003).

${ }^{22}$ C. Beekman, I. Komissarov, M. Hesselberth, and J. Aarts, Appl. Phys. Lett. 91, 062101 (2007).

${ }^{23} \mathrm{R}$. ÒHandley, Modern Magnetic Materials (Wiley and Sons, New York, 2000).

${ }^{24}$ J. Ye, Y. B. Kim, A. J. Millis, B. I. Shraiman, and P. Majumdar, and Z. Tesanovic, Phys. Rev. Lett. 83, 3737 (1999).

${ }^{25}$ W. E. Pickett and D. J. Singh, J. Magn. Magn. Mater. 172, 237 (1997).

${ }^{26}$ E. A. Livesay, R. N. West, S. B. Dugdale, G. Santi, and T. Jarlborg, J. Phys.: Condens. Matter 11, 1279 (1999).

${ }^{27}$ A. Chikamatsu, H. Wadati, H. Kumigashira, M. Oshima, A. Fujimori, M. Lippmaa, K. Ono, M. Kawasaki, and H. Koinuma, Phys. Rev. B 76, 201103 (2007).

${ }^{28}$ M. Shi, M. C. Falub, P. R. Willmott, J. Krempasky, R. Herger, L. Patthey, K. Hricovini, C. V. Falub, and M. Schneider, J. Phys.: Condens. Matter 20, 222001 (2008).

${ }^{29}$ R. S. Allgaier, Phys. Rev. 165, 775 (1968).

${ }^{30}$ M. Bibes, V. Laukhin, S. Valencia, B. Martínez, J. Fontcuberta, O. Yu. Gorbenko, A. R. Kaul, and J. L. Martínez, J. Phys.: Condens. Matter 17, 2733 (2005).

${ }^{31}$ M. Huijben, L. W. Martin, Y.-H. Chu, M. B. Holcomb, P. Yu, G. Rijnders, D. H. A. Blank, and R. Ramesh, Phys. Rev. B 78, 094413 (2008).

${ }^{32}$ A. Tebano, C. Aruta, S. Sanna, P. G. Medaglia, G. Balestrino, A. A. Sidorenko, R. De Renzi, G. Ghiringhelli, L. Braicovich, V. Bisogni, and N. B. Brookes, Phys. Rev. Lett. 100, 137401 (2008).

${ }^{33}$ J.-S. Lee, D. A. Arena, P. Yu, C. S. Nelson, R. Fan, C. J. Kinane, S. Langridge, M. D. Rossell, R. Ramesh, and C.-C. Kao, Phys. Rev. Lett. 105, 257204 (2010).

${ }^{34}$ C. Beekman, M. Porcu, H. W. Zandbergen, and J. Aarts, e-print arXiv:1102.4004.

${ }^{35}$ O. Ohtomo and H. Hwang, Nature (London) 427, 423 (2004). 